\title{
New cerebrospinal fluid biomarkers in Alzheimer's disease
}

\section{Future NEUROLOGY}
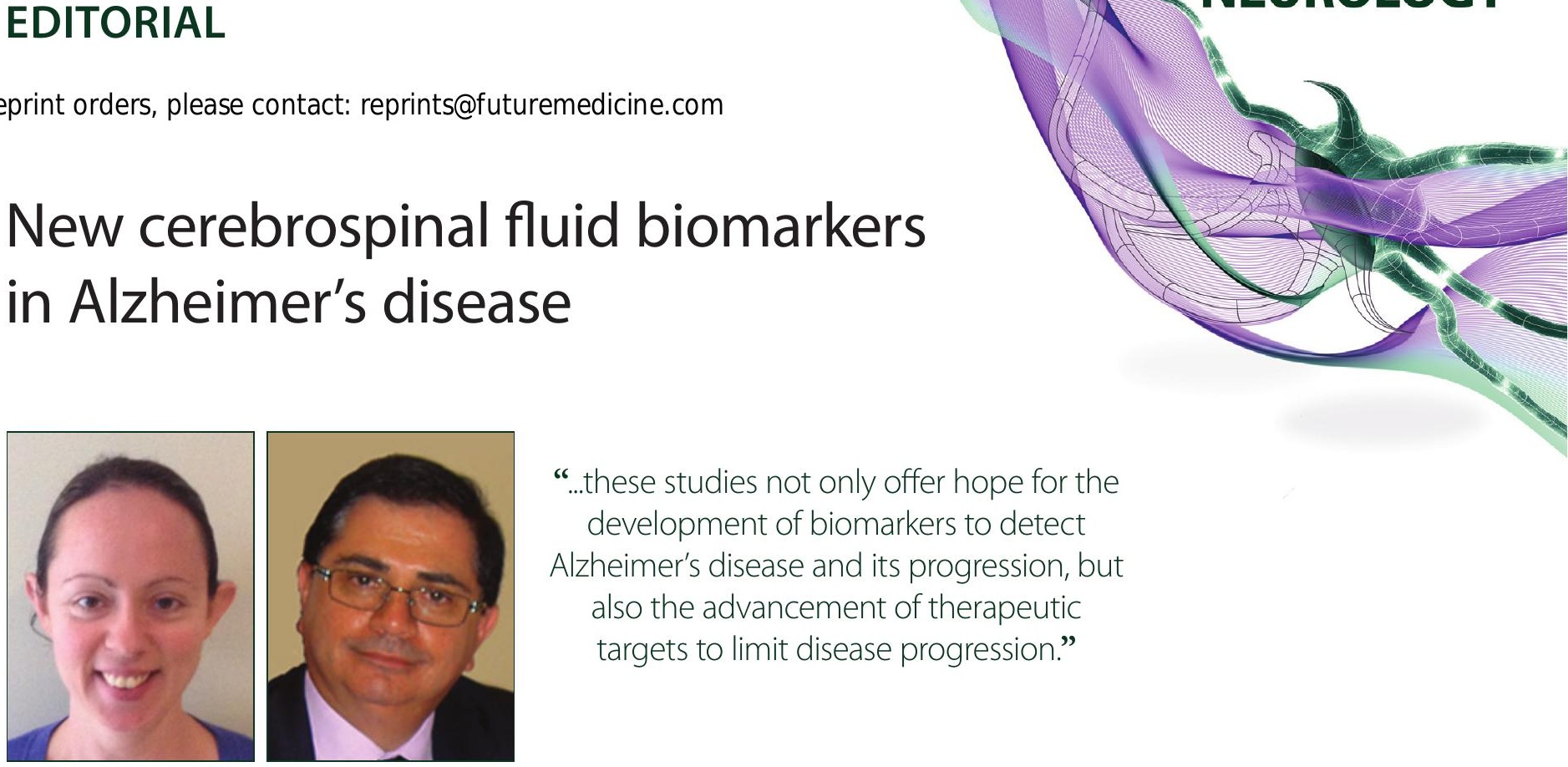

“...these studies not only offer hope for the development of biomarkers to detect

Alzheimer's disease and its progression, but also the advancement of therapeutic

targets to limit disease progression.”

Jennifer T Burchell' \& Peter K Panegyres*,1

First draft submitted: 7 March 2017; Accepted for publication: 20 March 2017; Published online: 5 May 2017

In 2015, we published a review on novel cerebrospinal fluid (CSF) biomarkers for detecting Alzheimer's disease (AD) [1]. We summarized the main potential CSF biomarkers as low amyloid $\beta_{1-42}\left(A \beta_{1-42}\right)$ and a high total tau (T-tau) and phosphorylated tau ( $\mathrm{p}$-tau). We discussed a number of other potential biomarkers, including those involving blood-brain barrier integrity (PDGFR- $\beta$ ), mitochondrial DNA (mtDNA), VEGF family members, calcium-sensor protein VILIP-1, $\beta$-site APP-cleaving enzyme 1 and the astrocyte marker YKL-40. More recently, a number of studies have focused on the potential use of other biomarkers such as TGF- $\beta$, neurofilament light polypeptide (NFL), autotaxin, $\alpha$-syn and complement. These modules offer promise for earlier detection of $\mathrm{AD}$ and other neurodegenerative disorders including Parkinson's disease (PD) and mild cognitive impairment (MCI).

\section{TGF- $\beta$}

TGF- $\beta$ are multifunctional cytokines that are secreted into the CSF to regulate the development of a number of cell types including the differentiation, proliferation and apoptosis of neuronal cells [2]. There are three different isoforms of TGF- $\beta$ in mammals: TGF- $\beta 1$, TGF- $\beta 2$ and TGF$\beta 3$. Increased levels of TGF- $\beta 1$ have been reported in lumbar CSF from AD patients [3] and in ventricular CSF from patients with PD [4], suggesting a possible use for TGB- $\beta 1$ as a biomarker for AD. TGF- $\beta 2$ has been reported to regulate $A \beta$-mediated neuronal death but it is unclear what precise role it plays in $\mathrm{AD}$ and Lewy body disease (LBD). Chong et al. [5] reported a significant increase in neocortical levels of TGF- $\beta 2$ in AD and LBD patients but not in PD patients. Interestingly, TGF- $\beta 2$ levels also correlated with neurofibrillary tangle scores, Lewy bodies (in the LBD group), dementia severity and soluble $A \beta 42$ concentration. This study demonstrates that TGF- $\beta 2$ is increased in the cortex of $\mathrm{AD}$ and dementia with Lewy bodies (DLB) patients and correlates with neuropathological and clinical markers of disease severity; and, therefore, has potential for use as a biomarker or as a target for pharmacological

'Neurodegenerative Disorders Research Pty Ltd, 4 Lawrence Avenue, West Perth, Western Australia 6005, Australia

*Author for correspondence: Tel.: +61 89481 6293; Fax: +61 89481 6294; research@ndr.org.au

\section{KEYWORDS}

- $\alpha$-synuclein - Alzheimer's disease

- autotaxin $\bullet$ complement

- dementia $\bullet$ mild cognitive impairment $\bullet$ neurofilament light

- Parkinson's disease • TGF- $\beta$ 


\section{“These results suggest that autotaxin could predict neuropathology and cognitive deficits in Alzheimer's disease.”}

approaches to AD and DLB. TGF- $\beta$ has been shown to be increased in CSF of patients with $\mathrm{PD}$ and $\mathrm{AD}$ but is thought to be an unreliable marker for other neurodegenerative disorders, such as amyotrophic lateral sclerosis, spinocerebellar degeneration and multiple system atrophy-cerebellar subtype. For this reason, it might be a helpful biomarker that could be used to distinguish between $\mathrm{AD}$ and $\mathrm{PD}$ from other neurodegenerative disorders.

\section{Neurofilament light polypeptide}

Neurofilaments are a group of heteropolymers found in the nervous systems and are responsible for the radial growth of axons during development, maintenance of axon caliber and neural transmission. One member of the family of neurofilaments is called neurofilament light polypeptide and is thought to correlate with axonal degeneration. CSF NFL levels have been reported to be associated with age [6], white matter lesions [7], $\mathrm{AD}[8]$ and other neurodegenerative diseases. A recent study of CSF NFL subunit levels in patients with MCI showed a positive correlation between NFL levels and hippocampal atrophy, suggesting that CSF NFL might offer promise as a marker for progression to $\mathrm{AD}[9]$.

The hippocampus is one of the areas of the brain with the highest atrophy rate in aging. In a study of 144 cognitively healthy older individuals, Idland et al. reported that NFL levels predicted neurodegeneration as measured by hippocampal atrophy rate independently of age, $\beta$-amyloid $1-42$ and $p$-tau [10]. They concluded that NFL was likely to be a biomarker of neurodegeneration independent of $\mathrm{AD}$ or age; however, further research is required to test the predictive value of NFL in the onset and progression of prodromal AD.

\section{Autotaxin}

Ectonucleotide pyrophosphatase/phosphodiesterase 2, also known as autotaxin, is produced by beige adipose tissue and acts to regulate energy metabolism. Autotaxin gene expression was increased in the frontal cortices of $\mathrm{AD}$ patients compared with non-AD patients, with a tendency for higher gene expression in patients with the ApoE e3/e4 genotype compared with the ApoE e3/e3 genotype [11]. Recently, elevated autotaxin levels have been found in the CSF of MCI and AD patients compared with healthy controls [12]. Interestingly, autotaxin was also positively correlated with levels of T-tau, p-Tau181, T-tau/A $\beta 1-42$ as well as p-Tau181/ A $\beta 1-42$, although to a lesser degree. These results suggest that autotaxin could predict neuropathology and cognitive deficits in AD.

\section{$\alpha$-syn}

$\alpha$-syn is involved in the regulation of synaptic plasticity, dopamine synthesis and neuronal differentiation. Abnormal accumulation of $\alpha$-syn in the brain is a pathological hallmark of PD and DLB, and can be found in the brains of individuals with AD. Therefore, there has been recent interest in developing an assay for the detection of $\alpha$-syn as a biomarker for neurodegenerative disorders. A simple ELISA-based method of detecting total $\alpha$-syn in CSF has recently been developed and validated using electrochemiluminescence technology in 50 CSF samples [13]. This assay offers promise for use as a biomarker not only in $\mathrm{AD}$, but also in other neurodegenerative disorders including PD and prion disease [14]. As $\alpha$-syn is found in other neurodegenerative conditions, its use as a marker to distinguish $\mathrm{AD}$ from other diseases is probably restricted.

\section{Complement}

Complement is an integral part of the innate immune system whose role is to recognize a target and opsonize it for engulfment and destruction by phagocytes. This is achieved by complement assembling the membrane attack complex which acts to create holes in a foreign pathogen's protective membrane. One major fault of this system is that complement can mistakenly attack host tissue which is known as bystander lysis. This mechanism can possibly contribute to the pathogenesis of $\mathrm{AD}$.

Three decades ago, it was discovered that complement could opsonize $\mathrm{A} \beta$ deposits in the brain of AD patients [15] and, soon after, it was reported that the membrane attack complex could attack dystrophic neurites [16]. The genes for C1q, C3 and C4 are expressed in the brain and have been reported to be upregulated more than threefold in AD [17]. Rogers et al. subsequently reported that $\mathrm{Clq}$ binds strongly to the $\mathrm{A} \beta$ peptide suggesting that the classical pathway could be activated by any tissue component recognized by complement [18]. Extracellular $\mathrm{A} \beta$ deposits in the brain have been reported to directly activate the complement system by associating with the opsonizing components 
of complement. Specifically, C1q can bind to $A \beta$ and its $\mathrm{N}$-terminal fragments which can potentially trigger the inflammatory cascade of AD [19]. Moreover, levels of complement components $\mathrm{C} 3$ and $\mathrm{C} 4$ and complement receptor 1 (CR1) have been reported to be elevated in the CSF of AD patients [20]. Daborg et al. reported that CSF levels of C3 and C4 were elevated in $\mathrm{AD}$ patients when compared with MCI patients who progressed to AD [20]. In addition, CSF levels of CR1 were elevated after combining MCI and $\mathrm{AD}$ groups compared with controls. These results, while promising, were deemed not useful for diagnostic purposes due to the small changes detected and could be strengthened by involving larger numbers of patients in future studies.

\section{Conclusion}

There have been some interesting and promising recent advances in detecting and validating potential CSF biomarkers for use in $\mathrm{AD}$ and other neurodegenerative disorders. A recent emphasis has been on immunological factors. TGF- $\beta$ is reported to be increased in the cortex of $A D$ and DLB patients and correlates with neuropathological and clinical markers of disease severity, and therefore has potential for use as a biomarker for AD. NFL could potentially be developed as a biomarker for neurodegeneration

which is independent of age or AD status; therefore, it might be helpful to distinguish AD from other neurodegenerative disorders. Recent studies report increased gene expression of autotaxin in the frontal cortex of $\mathrm{AD}$ patients and increased levels in the CSF of MCI and AD patients which could predict neuropathology and cognitive deficits in AD. $\alpha$-syn has been shown to be increased in the CSF of AD, PD and prion disease patients, and is, therefore, a useful marker for neurodegeneration but might not help in distinguishing these conditions. Other studies have demonstrated increased levels of complement C3 and C4, as well as CR1, in the CSF of AD patients. Taken together these studies not only offer hope for the development of biomarkers to detect $\mathrm{AD}$ and its progression, but also the advancement of therapeutic targets to limit disease progression.

\section{Financial \& competing interests disclosure}

This work was funded by Neurodegenerative Disorders Research Pty Ltd. The authors have no other relevant affiliations or financial involvement with any organization or entity with a financial interest in or financial conflict with the subject matter or materials discussed in the manuscript apart from those disclosed.

No writing assistance was utilized in the production of this manuscript.

Dis. 56(1), 157-166 (2016).

6 Vågberg M, Norgren N, Dring A et al. Levels and age dependency of neurofilament light and glial fibrillary acidic protein in healthy individuals and their relation to the brain parenchymal fraction. PLoS ONE 10(8), e0135886 (2015).

7 Sjögren M, Blomberg M, Jonsson M et al. Neurofilament protein in cerebrospinal fluid: a marker of white matter changes. J. Neurosci. Res. 66(3), 510-516 (2001).

3 Zetterberg H, Andreasen N, Blennow K. Increased cerebrospinal fluid levels of transforming growth factor- $\beta 1$ in Alzheimer's disease. Neurosci. Lett. 367(2), 194-196 (2004).

4 Mogi M, Harada M, Kondo T, Narabayashi H, Riederer P, Nagatsu T. Transforming growth factor- $\beta 1$ levels are elevated in the striatum and in ventricular cerebrospinal fluid in Parkinson's disease. Neurosci. Lett. 193(2), 129-132 (1995).

5 Chong JR, Chai YL, Lee JH et al. Increased transforming growth factor $\beta 2$ in the neocortex of Alzheimer's disease and dementia with Lewy bodies is correlated with disease severity and soluble A 442 load. J. Alzheimers
8 Olsson B, Lautner R, Andreasson U et al. CSF and blood biomarkers for the diagnosis of Alzheimer's disease: a systematic review and meta-analysis. Lancet Neurol. 15(7), 673-684 (2016).

9 Zetterberg H, Skillbäck T, Mattsson N et al. Association of cerebrospinal fluid neurofilament light concentration with Alzheimer disease progression. JAMA Neurol. 73(1), 60-67 (2016).

10 Idland AV, Sala-Llonch R, Borza T et al. CSF neurofilament light levels predict hippocampal atrophy in cognitively healthy older adults. Neurobiol. Aging 49, 138-144 (2017).
11 Umemura K, Yamashita N, Yu X et al. Autotaxin expression is enhanced in frontal cortex of Alzheimer-type dementia patients. Neurosci. Lett. 400(1-2), 97-100 (2006).

12 McLimans KE, Willette AA. Autotaxin as a CSF biomarker for central dysmetabolism and Alzheimer's disease outcomes. Alzheimers Dement. 12(7), 83 (2016).

13 FØrland MG, Öhrfelt A, Oftedal LS. Validation of a new assay for $\alpha$-synuclein detection in cerebrospinal fluid. Clin. Chem. Lab. Med. 55(2), 254-260 (2017).

14 Llorens F, Kruse N, Schmitz M et al. Evaluation of $\alpha$-synuclein as a novel cerebrospinal fluid biomarker in different forms of prion diseases. Alzheimers Dement. doi:10.1016/j.jalz.2016.09.013 (2016) (Epub ahead of print).

15 Eikelenboom P, Stam FC. Immunoglobulins and complement factors in senile plaques. Acta Neuropathol. 57(2-3), 239-242 (1982).

16 McGeer PL, Akiyama H, Itagaki S et al. Activation of the classical complement pathway in brain tissue of Alzheimer patients. Neurosci. Lett. 107(1), 341-346 (1989). 


\section{EDITORIAL Burchell \& Panegyres}

17 Walker DG, McGeer PL. Complement gene expression in human brain: comparison between normal and Alzheimer disease cases. Brain Res. Mol. Brain Res. 14(1-2), 109-116 (1992).

18 Rogers J, Cooper NR, Webster S et al. Complement activation by beta-amyloid in Alzheimer disease. Proc. Natl Acad. Sci. USA
89(21), 10016-10020 (1992).

19 Yasojima K, Schwab C, McGeer EG et al. Upregulated production and activation of the complement system in Alzheimer disease brain. Am. J. Pathol. 154(3), 927-936 (1999).
20 Daborg J, Andreasson U, Pekna M et al. Cerebrospinal fluid levels of complement proteins C3, C4 and CR1 in Alzheimer's disease. J. Neural Transm. (Vienna) 119(7), 789-797 (2012). 